nhất 01 tiểu muc có số điểm < 4,0 (đat 3,77 điểm). Điểm CLDV TB chung trong nghiên cứu của chúng tôi cao hơn so với những nghiên cứu khác như trong nghiên cứu của Al-Damen (2017) tai Jordan là 3,06 $\pm 0,82$.[9] Nghiên cứu của Kitapei tại Thổ Nhĩ Kỳ có điểm CLDV hữu hình cao nhất tương tự như nghiên cứu chúng tôi. Các nghiên cứu khác tiến hành trong nước của Lê Thị Kim Ngân và Lê Thị Thu Trang, Nguyễn Đăng Minh tiến hành trên các bệnh viện cấp độ khác nhau đều cho thấy CLDV yếu tố hữu hình là thấp nhất.

Trong số 10 tiểu mục khách hàng đánh giá cao hơn điểm trung bình chung (4,24 điểm) thì có 06 yếu tố thuộc về nhân viên y tế, kết quả này cho thấy bệnh viện đã rất quan tâm đến việc nâng cao chất lượng đội ngũ nhân viên y tế, thực hiện tốt chủ trương của ngành $Y$ tế trong việc đổi mới phong cách, thái độ phục vụ của nhân viên y tế hướng đến sự hài lòng người bệnh.

\section{KẾT LUẬN}

Kết quả nghiên cứu cho thấy người bệnh đánh giá tốt chất lượng dịch vụ tiêm nội nhãn do Bệnh viện Mắt Trung Ương cung cấp. Trong các yểu tố cấu thành nên chất lượng dịch vụ, yếu tố thuộc về NVYT được đánh giá cao từ người bệnh. Bệnh viên cần tiếp tục duy trì các biện pháp tăng cường chất lượng dịch vụ trong thời gian tới

\section{TÀI LIÊU THAM KHẢO}

1. J. Joseph Cronin JaSAT. Measuring Service Quality: A Reexamination and Extension. Journal of Marketing. 1992;56:55-68.

2. P Tan Le, G Fitzgerald (2014) 'Applying the SERVPERF Scale to Evaluate Quality of Care in Two Public Hospitals at Khanh Hoa Province, Vietnam'. Asia Pacific Journal of Health Management 9(2).

3. Advances in the Medical Treatment of Diabetic Retinopathy Diabetes Care [Internet]. [cited 2021 May 12]. Available from: https://care. diabetesjournals.org/content/32/8/1556.short

4. Multicenter trial of cryotherapy for retinopathy of prematurity. Preliminary results. Cryotherapy for Retinopathy of Prematurity Cooperative Group. Arch Ophthalmol. 1988 Apr;106(4):471-9.

5. Watkins PJ. Retinopathy. BMJ. 2003 Apr 26; 326(7395):924-6.

6. Mason JO, Nixon PA, White MF. Intravitreal Injection of Bevacizumab (Avastin) as Adjunctive Treatment of Proliferative Diabetic Retinopathy. American Journal of Ophthalmology. 2006 Oct 1;142(4):685-8.

7. Arab M Ghazi Tabatabaei S Rashidian A Rahimi Forushani A Zarei E (2012) 'The Effect of Service Quality on Patient loyalty a Study of Private Hospitals in Tehran, Iran-annotated-annotated'. Iranian Journa of Public Health, 41 (9) 71-77.

8. The CATT research group (2011) 'Ranibizumab and bevacizumab for Neovascular Aged-Related Macular Degeneration' The New England Journal of Medicine; 364:1897-1908.

9. Rula Al-Damen (2017) "Health Care Service Quality and Its Impact on Patient Satisfaction Case of Al-Bashir Hospital'"'. International Journal of Business and Management, 12 (9).

\title{
PHÂN TÍCH HÀNH VI MUA THỨC PHẨM CHỨC NĂNG CỦA NGƯờI TIÊU DÙNG TẠI THÀNH PHỐ HỒ CHÍ MINH
}

Nguyễn Thị Thu Sương², Nguyễn Thị Thu Phương1, Trần Thoại Khanh', Trương Văn Đạt ${ }^{1}$, Đặng Thị Kiều Nga ${ }^{1}$, Nguyễn Thị Hải Yến ${ }^{1}$

\section{TÓM TẮT}

Muc tiêu: Phân tích tình hình sử dung FF từ mô hình hành vi mua của người tiêu dùng tại Thành phố Hồ Chí Minh năm 2019. Phương pháp nghiên cứu: Nghiên cứu mô tả cắt ngang sứ dụng phương pháp đinh tính và định lương thông qua việc khảo sát người tiêu dùng tại các nhà thuốc trên địa bàn TPHCM có kinh doanh đa dạng TPCN năm 2018. Kết quả: Các yếu tố có tác động đến sự chấp nhận của người tiêu

${ }^{1}$ Đại học Y Dược Thành Phố Hồ Chí Minh ${ }^{2}$ Sở Khoa hoc và Công nghể Thành Phố Hồ Chí Minh Chịu trách nhiệm chính: Nguyễn Thị Hải Yến Email: haiyen@ump.edu.vn

Ngày nhận bài: 8.3.2021

Ngày phản biên khoa hoc: 27.4.2021

Ngày duyệt bài: 7.5.2021 dùng đối với TPCN bao gồm: (1) Thái độ, (2) Kiến thức, (3) Niềm tin, (4) Giá cả. Kết quả nghiên cứu cũng cho thấy "niềm tin" ảnh hưởng mạnh nhất đến mức đô chấp nhân của người tiêu dùng. Khi người tiêu dùng tin tưởng vào những lợi ích đối với sức khỏe mà TPCN có thể mang lai thì người tiêu dùng có khuynh hướng chấp nhận TPCN cao hơn. Kết luận: Lưu ý hành vi mua của người tiêu dùng trong quá trình phát triển và quảng bá sản phẩm của doanh nghiêp đến với người tiêu dùng cũng như trong các hoat đông tiếp thi của mình đảm bảo vì lợi ích sức khoẻ người tiêu dùng và phát triển thị trường TPCN.

Từ khóa: hành vi mua, thực phẩm chức năng, phân tích nhân tố, phân tích hồi quy tuyến tính, Thành phố Hồ Chí Minh

\section{SUMMARY}

ANALYSIS OF CONSUMER'S BUYING 


\section{BEHAVIOR OF FUNCTIONAL FOOD IN HO CHI MINH CITY}

Objectives: Develop a questionnaire to investigate the situation of using FF from the buying behavior model of consumers in Ho Chi Minh City in 2019. Method: This cross-sectional study used qualitative and quantitative methods through consumer interview at pharmacies in Ho Chi Minh City with a diversified FF business in 2019 . Result: The results show that 4 factors affecting consumer's acceptance of functional foods include: (1) Attitude, (2) Knowledge, (3) Belief in functional foods, (4) Perceived price. "Belief in functional foods" is shown to have the strongest impact on consumers' acceptance. When consumers believe in the health benefits that supplements can bring, consumers are more likely to accept functional foods. Conclusion: Pay attention to the buying behavior of consumers in the process of developing and promoting their products to consumers as well as raising discussions and recommendations on the management of marketing activities to increase the level of accepting functional foods for consumer health.

Key words: customer behavior, functional foods, Exploratory Factor Analysis, linear regression analysis, Ho Chi Minh city

\section{I. ĐĂT VẤN ĐỀ}

Kể từ khi xuất hiên, thực phẩm chức năng (TPCN) ngày càng phát triển với đây đủ các chủng loại, số lượng cũng như đi sâu về cơ chế, tác dụng đối với sức khỏe. Ở Việt Nam, đến năm 2015 đã có 1780 cơ sở kinh doanh TPCN với 3380 sản phẩm các loại, tỷ lệ người sử dụng TPCN đã tăng lên $40 \%$ ở người trưởng thành ${ }^{1}$. Thành phố Hồ Chí Minh (TPHCM) là một thành phố đông dân nhất cả nước, là thị trường tiềm năng để kinh doanh và phân phối các loại thực phẩm chức năng. Để có chiến lược phát triển và tiếp thị sản phẩm hiệu quả, tối đa hoá lợi nhuận, doanh nghiệp cần chú trọng việc nghiên cứu hành vi khách hàng, trong đó nổ lực để hiểu được tiến trình ra quyết định cưa người mua hàng, trên phương diện cá nhân lẫn tập thể. Nghiên cứu đặc điểm của cá nhân mua hàng, như nhân khẩu học hay tính cách và sự biến đổi trong hành vi mua hàng cốt Iõi để hiểu được mong muốn của mọi người ${ }^{2}$. Xuất phát từ những thực tế trên, nghiên cứu được thực hiện nhằm mục đích đưa ra câu trả lời về hành vi mua của NTD đối với các sản phẩm TPCN tại TPHCM, làm căn cứ khoa học để có thể phát triển chiến lược phù hợp.

\section{II. ĐỐI TƯợNG VÀ PHƯƠNG PHÁP NGHIÊN CỨU \\ 2.1. Thiết kế nghiên cứu. Nghiên cứu mô tả cắt ngang. \\ Thời gian thực hiện nghiên cứu là từ tháng 12/2018 đến tháng 05/2019.}

Quy trình thực hiện nghiên cứu được trình bày cụ thể ở Hình 1.

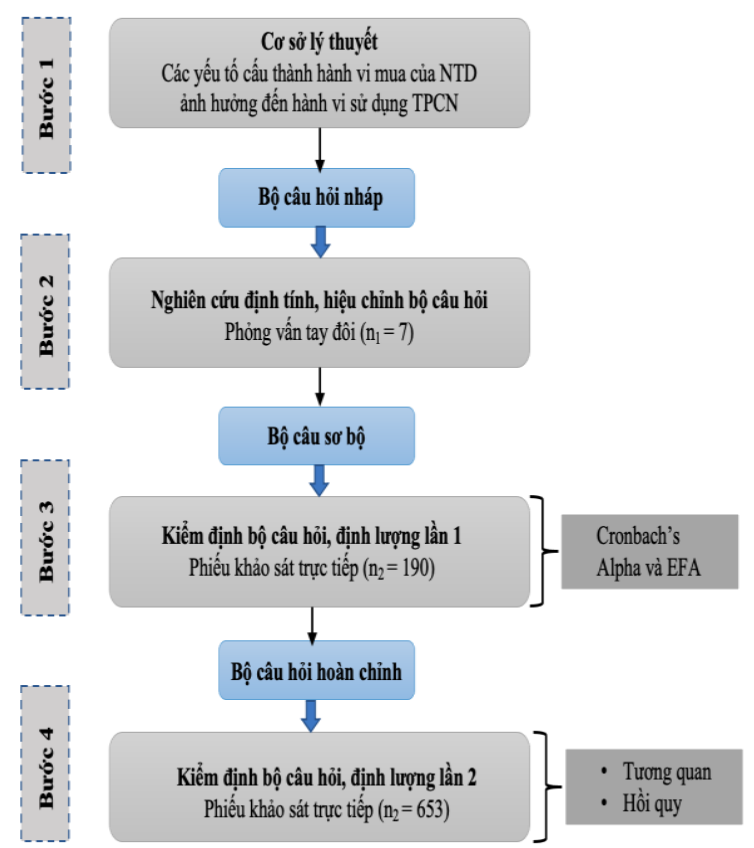

Hình 1. Quy trinh nghiên cứu của đề tài

2.2. Phương pháp nghiên cứu

2.2.1. Xây dựng bộ câu hỏi hành vi mua của người tiêu dung. Nghiên cứu thu thập các thành phần của hành vi mua từ (1) mô hình hành vi mua của NTD của Philip Kotler; (2) nghiên cứu được thực hiện trước đó về TPCN ${ }^{2-4}$. Từ đây, nghiên cứu tiến hành dịch thuật, tổng hợp và phỏng vấn với chuyên gia y tế, nhân viên tư vấn TPCN $\left(n_{1}=7\right)$ nhằm điều chỉnh nội dung cho phù hợp với tâm lý và hành vi của NTD. Nghiên cứu thu được thang đo bao gồm 28 câu hỏi (biến), tương ứng với 6 thành phần bao gồm: (1) thái độ của NTD (TD); (2) kiến thức về TPCN (KT); (3) niềm tin kiểm soát (NTKS); (4) niềm tin đối với TPCN (NT) (5) ảnh hưởng xã hội $(\mathrm{AH})$; (6) cảm nhận về giá cả (GC).

Tiếp theo, để đánh giá độ tin cậy của bộ câu hỏi, nghiên cứu tiến hành khảo sát với công thức cơ mẫu của K. A. Bollen ${ }^{5}$ :

$n_{\text {phiếu khảo sát }}=n_{\text {biến }} * 5+50$

Với thang đo có 28 biến (câu hỏi), cõ̃ mẫu tối thiểu phải là $28 * 5+50=190$.

Sau khi thu thập dữ liệu, nghiên cứu tiến hành loại bỏ các phiếu không hợp lệ do kết quả trả lời theo một trật tự nhất định hoặc không trả lời đủ các thông tin nghiên cứu. Nghiên cứu đánh giá độ tin cậy của thang đo sử dụng chỉ số Cronbach's Alpha, với điều kiện: (1) những biến quan sát có hệ số tương quan biến - tổng nhỏ 
hơn 0,3 sẽ bị loại và (2) giá trị Cronbach's Alpha từ 0,6 trở lên là đạt yêu cầu trong sử dụng. Để thu nhỏ và tóm tắt các dữ liệu, tiến hành phân tích nhân tố khám phá (EFA) thể hiện qua các chỉ số: (i) hệ số tải nhân tố (Factor loading) >0.3; (ii) hệ số Kaiser - Meyer - Olkin (0.5 s KMO $\leq 1$ ); (iii) Kiểm định Bartlett có ý nghĩa thống kê (Sig. <0.05) và (iv) phần trăm phương sai toàn bộ (Percentage of variance $>50 \%$ ).

2.2.2. Phân tích hành vi mua thức phẩm chức năng của người tiêu dùng TPHCM

Nghiên cứu sử dụng phương pháp lấy mẫu ngẫu nghiên NTA sử dụng TPCN tại TPHCM, do dân số TPHCM tính đến ngày 01/04/2019 là 8.993.082 người (>25.000 người), nên nghiên cứu sử dung công thức tính cỡ mẫu tối thiểu cho dân số vồ hạn định ${ }^{6}$ :

$$
\mathrm{n}=\frac{p(1-p) z_{1-\alpha_{d x}}}{d^{2}}
$$

Trong đó, n: Cõ̃ mẫu nghiên cứu

p: tỷ lệ ước tính do theo số liệu Cục An toàn thực phẩm đã điều tra (năm 2011) cho thấy hơn $43 \%$ dân số trên địa bàn TP.HCM đã từng sử dụng ít nhất một loại TPCN ${ }^{1}$

$Z^{2}(1-a / 2)$ : giá trị $z$ tương ứng với khoảng tin cậy mong muốn. Do đó nghiên cứu lựa chọn độ tin câyy $99 \%(a=0,01)$ nên $Z^{2}(1-a / 2)=2,58$.

d: độ chính xác tuyệt đối mong muốn, nghiên cứu lựa chọn $0,05(5 \%)$

Vì thế cõ̃ mẫu dự tính cho nghiên cứu sẽ được tính như sau:

$$
\mathrm{n}=\frac{p(1-p) z_{1-{ }^{2}} d_{2}}{d^{2}}=\frac{0,43(1-0,43) 2,58^{2}}{0,05^{2}}=653
$$

Nghiên cứu sử dụng mô hình gồm 6 biển với 28 mục câu hỏi từ cơ sở lý thuyết tương ứng với các thành phần hành vi mua TPCN gồm: (1) Thái đô của NTD (TD): đánh giá, cảm nhân của NTD về việc sử dụng TPCN; (2) Kiến thức về TPCN (KT): sự hiểu biến của NTD về lợi ích của TPCN đối với sức khoẻ, vai trò của việc bổ sung chất dinh dưỡng, tác dụng tích cực cũng như những tác dụng không mong muốn khi sử dụng TPCN; (3) Niềm tin kiểm soát (NTKS): việc sử dụng TPCN của NTD ảnh hưởng bởi các yéu tố như thu nhập, tình trạng sức khoẻ hiện tại, mối quan tâm về an toàn thực phẩm, mùi vị thực phẩm; (4) Niềm tin đối với TPCN (NT): sự tin tưởng vào ảnh hưởng tích cực của TPCN đối với sức khoẻ và việc kiểm soát các vấn đề liên quan đến sức khoẻ; (5) Ảnh hưởng xã hôii (AH): sự ảnh hưởng của việc sử dụng TPCN từ người thân, bạn bè, thái độ của người thân, bạn bè đối với việc sứ dụng TPCN; (5) Cảm nhận về giá cả (GC): cảm nhận của NTD về giá của TPCN, đánh giá của NTD về mức giá của TPCN so với hiệu quả mang lai; (6) Mức độ chấp nhận TPCN của NTD: phản ánh 02 trường hợp khác nhau NTD thực sự sử dụng hay sử dụng thử (trải nghiệm) đồng thời có thái độ tích cực với loại TPCN đó ${ }^{4}$.

Đồng thời, nghiên cứu đặt ra các giả thuyết về quan hệ giữa các thành phần của hành vi mua TPCN với mức độ chấp nhận TPCN của NTD: $\mathrm{H}_{1}$, $\mathrm{H}_{2}, \mathrm{H}_{3}, \mathrm{H}_{4}, \mathrm{H}_{5}, \mathrm{H}_{6}$ lân lượt là thái độ, kiến thức, niềm tin kiểm soát, niềm tin với TPCN, ảnh hưởng xã hội và cảm nhận về giá có quan hệ cùng chiều với mức độ chấp nhận TPCN của NTD.

Để kiểm đinh giả thuyết, nghiên cứu tiến hành phân tích tương quan và hồi quy để xây dựng phương trình hồi quy đa biến theo dạng

$$
\text { MĐCN }=\beta_{0}+\beta_{1} * \text { TD }+\beta_{2} * \text { KT }+\beta_{3} * \text { NTKS }
$$

$+\beta_{4} * \mathbf{N T}+\beta_{5} * \mathbf{A H}+\beta_{6} * \mathbf{G C}$

Hệ số $\beta$ là hệ số hồi quy riêng phần (Partial regression coefficients)

Kiểm định độ phù hợp của mô hình: (1) Giả thuyết $\mathrm{H}_{0}: \beta_{1}=\beta_{2}=\ldots=\beta i=0$; (2) Kiểm định sự phù hợp của mô hình sử dụng giá trị $F$ (kiểm định ANOVA) với độ tin cậy Sig. < 0,05.

\section{KẾT QUẢ NGHIÊN CỨU}

3.1. Bộ câu hỏi mô hình hành vi mua của người tiêuu dung. Dũ liêu được thu thâp thông qua hình thức phỏng vấn trực tiếp bằng bảng câu hỏi và hình thức trả lời qua email (trong đó hình thức trả lời qua email chiếm 63,15\% tương ứng với 120 phiếu). Sau khi kiểm tra mức độ tin cậy bằng Cronbach's Alpha thì có 5 biến bị loại (NTKS4, NT5, AH4, GC2, GC4), 23 biến quan sát còn laai đều có hệ số tương quan biến - tổng > 0,3 và hệ số tin cậy Cronbach's Alpha > 0,6 đạt yêu cầu nên tiếp tục được phân tích nhân tố khám phá (EFA). Qua 6 lần phân tích EFA, giá trị hê số tải nhân tố đã đạt đến mức tối đa theo từng biến và không gia tăng nữa, hơn nữa tổng phương sai trích luôn lớn hơn $60 \%$ ở cả 6 lần phân tích EFA. Các thành phần mới được kiểm định độ tin cậy lần nữa bằng Cronbach's Alpha, tất cả các biến đều có tương quan biến - tổng $>0,3$.

Như vậy, kết quả phân tích EFA cho thấy thang đo hành vi sử dụng TPCN từ 6 thành phần ban đầu được sắp xếp thành 5 thành phần gồm: (1) Thái độ của NTD, (2) Kiến thức về TPCN, (3) Niềm tin kiểm soát, (4) Niềm tin đối với TPCN, (5) Cảm nhận về giá. Danh sách các thành phần mới và kết quả Cronbach's Alpha của các thành phần mới của thang đo được thể hiện qua Bảng 1. 


\begin{tabular}{|c|c|c|}
\hline Mã & Nội dung & $\begin{array}{l}\text { Cronbach's } \\
\text { Alpha }\end{array}$ \\
\hline \multicolumn{3}{|c|}{ Thành phần 1- Thái độ của NTD (TD) } \\
\hline TD1 & Sử dụng TPCN là tốt & \multirow{5}{*}{0.854} \\
\hline TD2 & Sử dụng TPCN dể chịu, dể sử dụng & \\
\hline TD3 & Sứ dụng TPCN là hữu ích & \\
\hline TD4 & Việc sử dụng TPCN mang lại sự hài lòng & \\
\hline TD5 & Sử dụng TPCN thì tốt cho sức khoẻ & \\
\hline \multicolumn{3}{|c|}{ Thành phân 2 - Niềm tin đối với thực phẩm chức năng } \\
\hline NT1 & Mức độ an toàn của TPCN đã được nghiên cứu rất cẩn thận và toàn diện. & \multirow{5}{*}{0.746} \\
\hline NT2 & Tôi có thể cải thiện sức khỏe của mình bằng cách sử dụng TPCN. & \\
\hline NT3 & TPCN giúp tôi tự kiểm soát được sức khỏe của mình. & \\
\hline NT4 & TPCN dường như có ảnh hưởng tích cực đối với sức khỏe của tôi. & \\
\hline NT5 & Bạn bè của tôi thường sử dụng TPCN trong chế độ dinh dưỡng của mình. & \\
\hline \multicolumn{3}{|c|}{ Thành phân 3 - Kiến thức về TPCN (KT) } \\
\hline KT1 & Tôi hiểu rõ về các loại thực phẩm được bổ sung chất dinh dưỡng. & \multirow{3}{*}{0.724} \\
\hline KT2 & Tôi đánh giá cao kiến thức về TPCN của bản thân. & \\
\hline KT3 & Tôi biết TPCN có thể có tác dụng phụ. & \\
\hline \multicolumn{3}{|c|}{ Thành phân 4 - Niềm tin kiểm soát khi sử dụng TPCN (NTKS) } \\
\hline NTKS1 & Mức thu nhập của tôi có thể ảnh hưởng đên sự tiêu dùng TPCN của tôi. & \multirow{3}{*}{0.631} \\
\hline NTKS2 & Điều kiện sức khoẻ của tôi có thể ảnh hưởng đến việc tôi tiêu dùng TPCN. & \\
\hline NTKS3 & $\begin{array}{l}\text { Mối quan tâm về an toàn thực phẩm có thể ảnh hưởng đến việc sử dụng } \\
\text { TPCN của tôi. }\end{array}$ & \\
\hline \multicolumn{3}{|c|}{ Thành phân 5 - Cảm nhận về giá (GC) } \\
\hline GC1 & Tôi thấy TPCN quá đắt so với lợi ích sức khỏe được ghi nhận. & \multirow[b]{2}{*}{0.667} \\
\hline GC2 & $\begin{array}{l}\text { Tôi có nhiều lựa chọn tốt hơn so với việc mua TPCN có giá quá cao như' } \\
\text { hiện nay. }\end{array}$ & \\
\hline \multicolumn{3}{|c|}{ Thành phân 6 - Mức độ chấp nhận TPCN (CN) } \\
\hline CN1 & Tôi đã sử dụng thử TPCN, và dự định tiếp tục sử dụng loại sản phẩm này. & \multirow{4}{*}{0.88} \\
\hline CN2 & Tôi rất thích sử dụng TPCN trong chế độ dinh dưỡng của mình. & \\
\hline CN3 & Tôi đã (sẽ) giới thiệu người khác sử dụng TPCN. & \\
\hline CN4 & Tôi hài lòng với kết quả TPCN mà mình (đã) đang sử dụn & \\
\hline
\end{tabular}

Mô hình nghiên cứu được điêuu chỉnh lại để nghiên cứu quan hệ giữa các thành phần hành vi mua của NTD và mức độ chấp nhận TPCN của NTD với 5 thành phần mới và các giả thuyết $\mathrm{H}^{\prime} 1 ; \mathrm{H}^{\prime} 2 ; \mathrm{H}^{\prime} 3$; H'4; H'5 có quan hệ cùng chiều với mức độ chấp nhận TPCN của NTD thể hiện qua Hình 2.

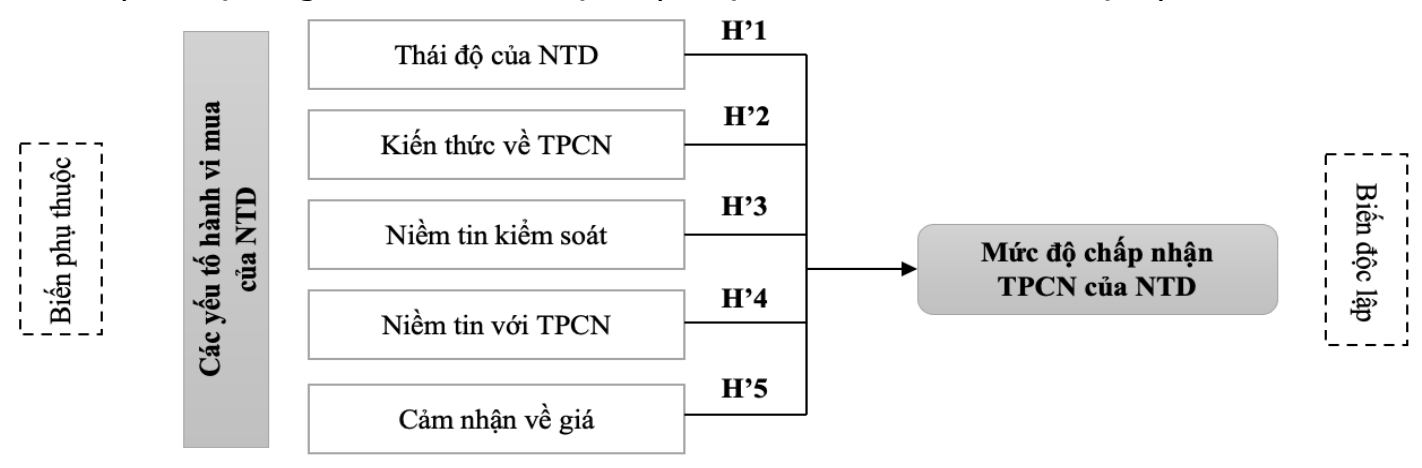

Hình 2. Mô hình nghiên cứu chính thức

3.2. Tình hình sử dụng thực phẩm chức năng của người tiêu dùng TPHCM

Nghiên cứu thu được 660 phiếu khảo sát, trong đó loại 26 phiếu không hợp lệ. Trong đó có 506 trường hợp sử dụng TPCN nên 506 phiếu này được chọn đưa vào phân tích. Đặc điểm nhân khẩu học của NTD tại TPHCM được thể hiện qua Bảng 2. 
VIETNAM MEDICAL JOURNAL N01 - MAY - 2021

Bảng 2. Đặc điểm nhân khẩu học và tình hình sử dụng TPCN của NTD tại TPHCM $(n=560)$

\begin{tabular}{|c|c|c|c|}
\hline Biến kiếm soát & Số lương (\%) & Đăc điếm sứ dung & Ty̆ lệ \% \\
\hline \multicolumn{2}{|l|}{ Giới tính } & \multicolumn{2}{|l|}{ Theo mục đích sử dụng } \\
\hline Nam & $207(40,91)$ & Hô trợ điều trị & 48,42 \\
\hline Nữ & $299(59,09)$ & Giảm béo & 37,55 \\
\hline \multicolumn{2}{|l|}{ Tuối } & Làm đep & 35,18 \\
\hline $15-30$ tuối & $321(63,44)$ & Tăng cường miên dịch & 32,81 \\
\hline $31-45$ tuối & $107(21,15)$ & Phòng bệnh & 32,21 \\
\hline $46-60$ tuối & $62(12,25)$ & Hô trợ tiêu hoá & 30,04 \\
\hline Trên 60 tuối & $16(3,16)$ & Chống lão hoá & 23,91 \\
\hline Trình độ & & Tăng cương sinh lực & 14,62 \\
\hline Trung học phố thông & $69(13,64)$ & Giảm cholesterol & 12,85 \\
\hline Cao đằng trung cấp & $123(24,31)$ & Hạ huyết áp & 11,86 \\
\hline Đại học & $263(51,98)$ & Dưỡng não, bố não & 11,46 \\
\hline Sau đại học & $51(10,08)$ & \multicolumn{2}{|c|}{ Theo nguôn thông tin tham khảo } \\
\hline \multicolumn{2}{|c|}{ Thu nhập bình quân hàng tháng } & Nhân viên y tế & 59,09 \\
\hline Dưới 5 triệu & $244(48,22)$ & Gia đình, bạn bè, người thân & 39,53 \\
\hline Từ 5- 10 triệu & $150(29,64)$ & Internet & 33,00 \\
\hline Từ $10-18$ triệu & $71(14,03)$ & Sách báo, tạp chí & 21,34 \\
\hline Từ 18-32 triệu & $19(3,75)$ & Truyền hình & 10,08 \\
\hline Trên 32 triệu & $20(3,95)$ & Theo địa điếm mua TPCN & \\
\hline \multicolumn{2}{|c|}{ Nghề nghiệp } & Nhà thuốc & 77,67 \\
\hline Sinh viên & $191(37,75)$ & Cửa hàng bán lẻ, siêu thị & 20,95 \\
\hline Viên/Công chức nhà nước & $48(9,49)$ & Online & 20,36 \\
\hline Nhân viên văn phòng & $88(17,39)$ & Mua trực tiếp & 14,23 \\
\hline Kinh doanh & $82(16,21)$ & & \\
\hline Nghề nghiệp khác & $97(19,17)$ & & \\
\hline \multicolumn{2}{|c|}{ Tình trạng hôn nhân } & & \\
\hline Độc thân & $\begin{array}{l}333(65,81) \\
173(34,19)\end{array}$ & & \\
\hline
\end{tabular}

Kết quả khảo sát đặc điếm hành vi mua theo tiến trình ra quyết định mua TPCN của NTD được thể hiện qua Bảng 3.

Bước đâu tiên khi tiến hành phân tích hồi quy tuyến tính bội là xem xét mối tương quan tuyến tính giữa tất cả các biến. Kết quả cho thấy nếu xét theo mối quan hệ độc lập và không bị ảnh hưởng bởi các thành phần khác thì khi mỗi thành phần biến độc lập trong mô hình nghiên cứu tăng thì kéo theo thành phần biến phụ thuộc là mức độ chấp nhận TPCN của NTD cũng tăng theo. Do đó, tất cả 5 thành phần đều tồn tại trong mô hình nghiên cứu và mô hình hồi quy đa biến có thể sử dụng để xem xét mối quan hệ tuyến tính giữa các biến độc lập và biến phụ thuộc. Tuy nhiên, giá trị tuyệt đối của các hệ sổ tương quan giữa các biến độc lập dao động từ 0.133 đến 0.345 . Điều này cho thấy một số biến độc lập có mối tương quan với nhau, có thể gây ra hiện tượng đa cộng tuyến.

Ma trận hệ sổ tương quan Pearson được dùng để kiểm tra mối quan hệ tuyến tính giữa từng biến độc lập với biến phụ thuộc và giữa các biến độc lập với nhau thể hiện quan Bảng 4.

Bảng 4. Ma trân tương quan giữa các biến

\begin{tabular}{|c|c|c|c|c|c|c|c|}
\hline \multicolumn{2}{|c|}{ Thành phân } & TD & KT & NTKS & NT & GC & MĐCN \\
\hline TD & $\stackrel{r}{r}$ & 1 & & & & & \\
\hline KT & $\begin{array}{c}r \\
\text { Sig. }\end{array}$ & $\begin{array}{c}\mathbf{0 , 1 6 9} \\
0,000\end{array}$ & 1 & & & & \\
\hline NTKS & $\begin{array}{c}\mathbf{r} \\
\text { Sig. }\end{array}$ & $\begin{array}{c}\mathbf{0 , 0 9 1} \\
0,000\end{array}$ & $\begin{array}{r}\mathbf{0 , 2 3 7} \\
0,000\end{array}$ & 1 & & & \\
\hline NT & $\begin{array}{c}\mathbf{r} \\
\text { Sig. }\end{array}$ & $\begin{array}{c}\mathbf{0 , 3 4 5} \\
0,000\end{array}$ & $\begin{array}{c}\mathbf{0 , 3 3 9} \\
0,000\end{array}$ & $\begin{array}{c}\mathbf{0 , 2 8 9} \\
0,000\end{array}$ & 1 & & \\
\hline GC & $\begin{array}{c}\mathbf{r} \\
\text { Sig. }\end{array}$ & $\begin{array}{c}\mathbf{- 0 , 1 8 8} \\
0,000\end{array}$ & $\begin{array}{l}\mathbf{0 , 0 1} \\
0,000\end{array}$ & $\begin{array}{c}\mathbf{0 , 0 9 3} \\
0,000\end{array}$ & $\begin{array}{c}\mathbf{- 0 , 1 3 3} \\
0,000\end{array}$ & 1 & \\
\hline MĐCN & $\stackrel{r}{\mathbf{r}}$ & $\begin{array}{c}\mathbf{0 , 3 1 9} \\
0,000\end{array}$ & $\begin{array}{c}\mathbf{0}, 337 \\
0,000\end{array}$ & $\begin{array}{c}\mathbf{0 , 1 4 8} \\
0,000\end{array}$ & $\begin{array}{r}\mathbf{0 , 5 1 7} \\
0,000\end{array}$ & $\begin{array}{c}\mathbf{0 , 1 9 6} \\
0,000\end{array}$ & 1 \\
\hline
\end{tabular}


Các giá trị in đậm là Hệ số tương quan.

Các giá trị dưới là Mức ý nghĩa (2- đuôi) của tương quan. Kết quả phân tích hồi quy tuyến tính bội với biến phụ thuộc mức độ chấp nhận TPCN và 5 biến độc lập (thái độ, kiến thức, niềm tin kiểm soát, niềm tin, cảm nhận về giá) cho thây biến niềm tin kiểm soát có Sig. $=0,38$ (> $0,50)$ nên biến bị loại. Nghiên cứu tiếp tục phân tích hồi quy với 4 biến, gồm: thái độ, kiển thức, niềm tin, giá cả.

Kết quả phân tích hồi quy được thể hiện qua Bảng 5 . Mô hình có giá trị $\mathrm{R}^{2}$ hiệu chỉnh bằng
0,3741; tương ứng với 37,41\% sự biến thiên của mức độ chấp nhận TPCN được giải thích bởi 4 thành phân của hành vi mua. Phân tích ANOVA cho thấy $\mathrm{F}$ có mức ý nghĩa Sig. = 0,000 (< 0,050 ) có nghĩa là mô hình hồi quy phù hợp với dữ liệu thu thâp được và các biến đưa vào đều có ý nghĩa thổng kề. Giá trị hệ số VIF của các biến đều nhỏ hơn 2, do đó, mô hình hồi quy không vi phạm hiên tượng đa cộng tuyến? hình hồi quy đa biến có dạng: $\mathbf{M Đ C N}=\mathbf{0 , 6 0}+$ $0,16 * T D+0,20 * K T+0,40 * N T-0,14 * G C$

Bảng 5. Kết quả phân tích hồi quy đa biến

\begin{tabular}{|c|c|c|c|c|c|c|c|}
\hline \multicolumn{8}{|c|}{ Hệ số (Coefficients) } \\
\hline \multirow{2}{*}{ Biến } & \multicolumn{2}{|c|}{$\begin{array}{c}\text { Hêe số } \\
\text { chưa chuẩn hóa }\end{array}$} & \multirow{2}{*}{$\begin{array}{c}\begin{array}{c}\text { Hê số } \\
\text { chuấn hóa }\end{array} \\
\text { Beta } \\
\end{array}$} & \multirow[t]{2}{*}{$\mathbf{t}$} & \multirow{2}{*}{ Sig. } & \multirow{2}{*}{ Tolerance } & \multirow[t]{2}{*}{ VIF } \\
\hline & B & Đô lêch chuẩn & & & & & \\
\hline (Hằng số) & 0.60 & 0.22 & & 2.77 & 0.01 & & \\
\hline Thái độ của NTD & 0.17 & 0.04 & 0.16 & 4.67 & 0.00 & 0.86 & 1.16 \\
\hline Kiến thức về TPCN & 0.25 & 0.04 & 0.20 & 5.81 & 0.00 & 0.85 & 1.17 \\
\hline Niềm & 0.50 & 0.05 & 0.40 & 10.99 & 0.00 & 0.76 & 1.31 \\
\hline Cảm nhân về giá & -0.13 & 0.03 & -0.14 & -4.53 & 0.00 & 0.98 & 1.02 \\
\hline
\end{tabular}

\section{BÀN LUẬN}

Một điểm mới của nghiên cứu là xây dựng bô câu hỏi điều tra tình hình sử dụng TPCN từ mổ hình hành vi mua của NTD tại TPHCM. Với nội dung này, nghiên cứu đi vào tìm hiểu toàn bộ các yếu tố tác động đến hành vi mua của NTD. Các yếu tố này bao gồm các yếu tố kích thích từ bên ngoài, những suy nghĩ, cảm nhận diễn ra trong hộp đen ý thức của NTD và những phản ứng đáp lại của NTD. Nghiên cứu cũng tiến hành phân tích tình hình sử dụng TPCN tại TPHCM thông qua mô hình hồi quy, kết quả cho thây "niềm tin với TPCN" ảnh hưởng mạnh nhất đến mức độ chấp nhận của NTD. Đây chính là một điểm rất quan trọng mà những doanh nghiệp sản xuất và/hoặc kinh doanh trong lĩnh vực TPCN cần lưu ý trong quá trình phát triển và quảng bá sản phẩm của mình đến với NTD. Các giải pháp sau đây được đề xuất để gia tăng mức độ chấp nhận TPCN của NTD: (1) Nâng cao ý thức của NTD về TPCN: không chỉ các chuyên gia như bác sĩ mà cả NTD ngày càng nhận thức rõ mỗi quan hệ mật thiết giữa dinh dưỡng và tình trạng sức khoé ${ }^{8}$. Vì vậy, các doanh nghiệp cần gửi một thông điệp rõ ràng trong kế hoạch truyền thông của mình rằng TPCN là loại thực phẩm với các dưỡng chất được bổ sung, tăng cường nhằm đáp ứng cho các mục đích dinh dưỡng nhất định, cung cấp dưỡng chất cần thiết, tăng sức đề kháng và giảm bớt nguy cơ bệnh tật; (2) Xây dựng niềm tin của NTD bằng cách hoàn thiệ khung pháp lý cho sản xuất và kinh doanh TPCN: ngày càng nhiều hiện tượng tiêu cực với mức độ ngày một gia tăng đang diễn ra trên thị trường TPCN như thông tin trên bao bì không chính xác hoăc bi thổi phông, sản phẩm chưa được kiểm duyệt và công nhận, quản cáo sai về nội dung so với tiêu chuẩn cồng bố, hàng giả, hang nhái hoặc hàng không rõ nguồn gốc... Điều này dẫn đến sự mất lòng tin của NTD. Vì vậy, các doanh nghiệp cần phối hợp với cớ quan chức năng để xây dựng các quy định pháp luật, các tiêu chuẩn, quy chuẩn và quy định quản lý liên quan để bảo vệ lợi ích của chính doanh nghiệp, tạo lập và củng cố niềm tin cho NTD; (3) "Kiến thức về TPCN" cũng tác động không nhỏ đến mức độ chấp nhận của NTD. Càng hiểu rõ về các thành phần cũng như tác động của chúng đến sức khoẻ thì NTD càng có khuynh hướng sử dụng TPCN nhiều hơn. Vì vậy, các doanh nghiệp cần xây dựng kế hoach cu thể đem kiến thức về TPCN đến gần hơn với NTD. Cách thức thực hiện cũng cần phải linh hoạt, đa dạng, thường xuyên, nội dung dễ hiểu, hấp dẫn, phong phú, phối hợp nhiều hình thức.

Cõ mẫu của nghiên cứu ở bước đánh giá bảng câu hỏi và thang đo tuy đáp ứng đủ số lượng theo tiêu chí kiểm định Cronbatch's Alpha và EFA là 190 người nhưng chưa thể đại diện cho toàn bộ dân số do đối tượng chủ yếu là sinh viên và nhẩn viên văn phòng. Ơ bước khảo sát và phân tích tình hình sử dụng TPCN thông qua 
bô câu hỏi xây dựng từ hành vi mua TPCN của người tiêu dùng tại TPHCM xây dựng được, với cõ̃ mẫu lớn hơn nhiêu so với các đề tài trước đây là 506 người, dân số mang tính đại diện với đầy đủ các nhóm tuổi, nhóm ngành nghề, tuy nhiên, việc lựa chọn mẫu còn chưa thực sự chặt chẽ, cần thực hiện chọn mẫu cụ thể và rổ ràng hơn. So với nghiên cứu của tác giả Nguyễn Thị Thu Hà, 2015 (287 người chưa từng sử dụng TPCN ở Đà Nẵng) và Hoàng Thị Phương Thảo, 2016 (300 người đã từng mua và/hoặc sử dụng TPCN tại TPHCM), cõ mẫu của nghiên cứu này lớn hơn và tập trung hơn vào người tiêu dùng tại TPHCM nên đã cung cấp được thông tin cập nhật trong dữ liệu nghiên cứu thị trường cho doanh nghiêp - đây là nguồn tham khảo chi tiết và cập nhật hơn khi các doanh nghiệp muốn tìm hiểu và phát triển sản phẩm TPCN ${ }^{3,4}$.

Tuy nhiên, nghiên cứu chỉ mới dừng lại ở bước xây dựng bộ câu hỏi điều tra tình hình sử dụng TPCN của NTD tại TPHCM (nhân khẩu học, mục đích sử dụng TPCN, nguồn tìm kiếm thông tin của NTD, kiến thức, thái độ, niềm tin, cảm nhận về giá của NTD về TPCN...). Vì vậy, cần có các nghiên cứu phân tích sâu hơn về ảnh hưởng của các yếu tố này đến mức độ chấp nhận và sử dụng TPCN. Hơn nữa, các nghiên cứu thị trường chỉ đúng trong khoảng thời gian tiến hành nghiên cứu, doanh nghiệp cần tiến hành thường xuyên nếu muốn hiểu rõ̃ thị hiếu NTD từ đó đề ra chiến lược kinh doanh phù hợp nhất trong từng khoảng thời gian nhất định.

\section{KẾT LUÂN}

Trong bối cảnh TPCN ngày càng phát triển thì cơ hội đâuu tư cho các doanh nghiệp ngày càng rộng mở, đồng thời cũng là thách thức trong việc quản lý của cơ quan chức năng. Bộ câu hỏi hoàn thiện đã hỗ trợ cung cấp thông tin đầy đủ, khách quan cho các doanh nghiệp trong quá trình nghiên cứu thị trường, đồng thời giúp nhà quản lý có cái nhìn toàn diên trong công tác quản lý về việc sử dụng TPCN của NTD. Cả 4 thành phần: (1) Thái độ, (2) Kiến thức, (3) Niềm tin, (4) Giá cả đều ảnh hưởng rất lớn đến sự chấp nhận TPCN của NTD, không thể xem nặng hay xem nhẹ bất kỳ thành phần nào, đây cũng là một trong những cơ sở để đưa ra các giải pháp đối với các doanh nghiệp trong việc thay đổi hành vi sử dụng TPCN của người tiêu dùng.

\section{TÀI LIẸU THAM KHẢO}

1. Trân Đáng (2014), Số liêu thị trường TPCN 2000 - 2013, Hiệp hội Thực Phẩm Chức Năng.

2. Kotler P. et al. (2012), Marketing management 14th Edition, Pearson Prentice Hall, Upper Saddle River, NJ, pp. 98-118.

3. Nguyễn Thị Thu Hà (2015), Nghiên cứu các yếu tổ ảnh hưởng đến ý định mua thực phẩm chức năng của ngưới tiêu dùng tại Đà Nẵng, luận văn thạc sĩ, trường đại học Đà năng, thành phố Đà Nẵng.

4. Hoàng Thị Phương Tháo (2016), Mức độ chấp nhận thực phẩm chức năng của người tiêu dùng, tạp chí đại học mở thành phố Hồ Chí Minh.

5. K. A. Bollen (1989), Structural equations with latent variables, Wiley, New York.

6. Tổng điêuu tra dân số và nhà ở trung ương (2019), Tổng điều tra dân số và nhà ở năm 2019, Tổng Cục Thống kê, http://tongdieutradanso.vn/ tphcm-cong-bo-ket-qua-so-bo-tong- dieu-tra-danso-va-nha-o-nam-2019.html, ngày truy cập 23/04-2020.

7. Hair, J.F., Black, W.C., Babin, B.J., \& Anderson, R.E. (2010). Multivariate Data Analysis. Seventh Edition. Prentice Hall, Upper Saddle River, New Jersey.

8. Menrad, K. (2003) Market and Marketing of Functional Food in Europe. Journal of Food Engineering, 56, 181-188.

\section{NGHIÊN CỨU TÁC DỤNG CHỐNG VIÊM THỰC NGHIỆM CỦA VIÊN NANG HỖ TRỢ ĐIỀU TRI ECZEMA}

\section{TÓM TẮT}

Mục tiêu: Đánh giá được tác dụng chống viêm của viên nang hỗ trợ điêuu trị eczema trên chuột cống trắng. Đối tượng và phương pháp: Viên nang hô

\author{
${ }^{1}$ Trường Đai hoc Dước Hà Nôi \\ Chịu trách nhiệm chính: Nguyễn Mạnh Tuyển \\ Email: tuyennm@hup.edu.vn \\ Ngày nhận bài: 4.3.2021 \\ Ngày phản biên khoa hoc: 22.4.2021 \\ Ngày duyệt bài: 4.5.2021
}

\section{Phạm Thái Hà Văn ${ }^{1}$, Nguyễn Mạnh Tuyển ${ }^{1}$}

trợ điều trị eczema được bào chế từ cao chiết ethanol của bài thuốc hỗ trợ điều trị eczema. Đánh giá tác dụng chống viêm cấp theo phương pháp gây tràn dịch màng bụng chuột bằng carrageenin; tác dụng chống viêm mạn theo phương pháp gây u hạt thực nghiệm. Kết quả: Viên nang hố trợ điều tri eczema có tác dụng chống viêm cấp ở chuột cống trẳng trên mô hình gẩy viêm phúc mac ở liều tương đương lâm sàng $0,6 \mathrm{~g} / \mathrm{kgTT}$ và $1,8 \mathrm{~g} / \mathrm{kg} T$. Trên mô hình gây u hạt thực nghiệm, viên nang hố trợ điều trị eczema có tác dụng chống viêm mạn ở liểu tương đương lâm sàng $0,6 \mathrm{~g} / \mathrm{kg} T \mathrm{~T}$ và $1,8 \mathrm{~g} / \mathrm{kgTT}$, tác dụng này tương đương 